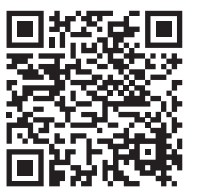

Palabras clave: Simulación, punción lumbar, competencia, residentes, pediatría.

Keywords: Simulation, lumbar puncture, competence, residents, pediatrics.

* Médico residente de Pediatría.

‡ Médico traumatólogo,

Magíster en

Investigación en

Ciencias de la

Salud.

Pontificia

Universidad

Católica de Chile.

Recibido: 05/02/2021

Aceptado: 24/03/2021

doi: $10.35366 / 99865$

\section{Concordancia entre competencia autorreportada y desempeño en punción lumbar simulada en residentes de un programa de formación en pediatría}

\author{
Concordance between self-reported competence \\ and simulated lumbar puncture performance in \\ residents of a pediatric training program
}

María José Pezzani,* Pablo Besa ${ }^{\ddagger}$

\section{RESUMEN}

Introducción: La punción lumbar es uno de los procedimientos clínicos invasivos más comúnmente realizados por un pediatra, por lo que The Royal College of Physicians and Surgeons of Canada y el Accreditation Council for Graduate Medical Education, sugieren que las experiencias educacionales deben asegurar la adquisición de competencias en aquel procedimiento. Muchos estudios han demostrado la falta de entrenamiento formal y la baja exposición a dicho tipo de punción durante la práctica clínica, razón por la cual muchos médicos están en riesgo de completar su residencia con una técnica y práctica inadecuadas del procedimiento. Objetivos: Determinar la capacidad que tienen los residentes de Pediatría al final de su formación a fin de realizar una punción lumbar en un ambiente simulado y comparar su desempeño con su autopercepción de competencias. Material y métodos: Se reclutó a todos esos médicos de último año de un programa universitario de Pediatría de tres años de duración. Se aplicó una encuesta en línea donde se preguntaron tres puntos, entre ellos la autopercepción de competencias en la realización de una punción lumbar basada en una escala de evaluación global ya validada. Posteriormente, los residentes realizaron tal clase de punción en el Centro de Simulación de la Pontificia Universidad Católica de Chile. Los vídeos de cada participante fueron asignados con un número al azar para su posterior análisis por parte de dos evaluadores expertos, de forma independiente, basados en la escala de valoración global con que se midió la autopercepción de competencia en la encuesta aplicada a dichos médicos. La correlación entre observadores se realizó mediante el coeficiente weighted Kappa (wK). Resultados: Once residentes completaron la evaluación. En la autopercepción, todos los alumnos se consideraron competentes ( 7 de 11 ) o principiantes (4 de 11). Los evaluadores expertos consideraron a la mayoría de los participantes como novatos (19 de 22) y pocos principiantes

\section{ABSTRACT}

Introduction: Lumbar puncture is one of the most common invasive clinical procedures performed by a pediatrician, so The Royal College of Physicians and Surgeons of Canada and the Accreditation Council for Graduate Medical Education suggest that educational experiences must Many studies have shown a lack of formal training and low exposure to said puncture during clinical practice, so many physicians are at risk of completing their residency with inadequate technique and practice of the procedure. Objectives: To determine the capacity of Pediatric residents at the end of their training to execute a lumbar performance with their self-perception of skills. Material and methods: All last-year physicians of a three-year university Pediatrics program were recruited. An online survey was applied where three questions were asked, including the self-perception of competencies in executing a lumbar puncture based on a global evaluation scale already validated. Subsequently, these doctors performed such puncture at the Simulation Center of the Pontificia Universidad Católica de Chile. The videos of each resident were assigned a random number for subsequent analysis by two expert evaluators independently, based on the global evaluation scale with which self-perception residents. The inter-observer correlation was performed using the weighted Kappa ( $w K$ ) coefficient. Results: Eleven of them completed the evaluation. In self-perception, all students considered themselves competent ( 7 out of 11) or beginners (4 out of 11). The expert evaluators considered most of the participants as novices (19 of 22) and a few beginners (3 of 22), with substantial agreement among both experts ( $w K 0.62 ; p<0.01$ ). All the students who were evaluated as beginners perceived themselves as ensure the acquisition of competencies in that procedure. puncture in a simulated environment and to compare their of competence was measured in the survey applied to the

Citar como: Pezzani MJ, Besa P. Concordancia entre competencia autorreportada y desempeño en punción lumbar simulada en residentes de un programa de formación en pediatría. Rev Latinoam Simul Clin. 2021; 3 (1): 22-27. https://dx.doi.org/10.35366/99865 
(3 de 22), existiendo un acuerdo sustancial entre los evaluadores (wK 0.62; $\mathrm{p} 0.01$ ). Todos los estudiantes que fueron evaluados a nivel de principiantes se autopercibieron como competentes. El acuerdo entre la percepción del residente y los evaluadores fue escaso (wK 0.00; $\mathrm{p}<0.01$ ). El promedio de punciones lumbares realizadas durante los tres años de residencia arrojó un valor de 11.41. De estos médicos, el 58\% nunca había realizado semejante punción simulada previamente. Conclusiones: Los residentes se autoevalúan más competentes de lo que realmente son. Además, existe una gran variabilidad en cuanto a la exposición a la punción lumbar a lo largo de la residencia, asociado a un escaso entrenamiento del procedimiento en simulación. competent. The agreement between the perception of the resident and the evaluators was poor ( $w K 0.00 ; p<0.01$ ). The average number of lumbar punctures accomplished during the three years of residence averaged 11.41. Of the residents, 58\% had never performed a simulated lumbar puncture previously. Conclusions: These physicians assess themselves more competent than they really are. In addition, there is a great variability in terms of exposure to said puncture during residency, associated with little training in the simulation procedure.

\section{INTRODUCCIÓN}

T a punción lumbar (PL) es uno de los pro$\amalg$ cedimientos clínicos invasivos más comúnmente realizados por un pediatra. ${ }^{1}$ Un estudio descriptivo realizado en Estados Unidos en 2018, a partir del análisis de la base de datos National Emergency Department Survey (NEDS), determinó que en el transcurso del año 2010 se realizaron 362,718 punciones lumbares diagnósticas en Servicios de Urgencia, 89,106 (25\%) fueron en pacientes pediátricos, siendo la mayoría niños menores de cinco años. ${ }^{2}$

Dicha forma de punción es una importante herramienta diagnóstica y terapéutica que se realiza con el objetivo de obtener una muestra de líquido cefalorraquídeo (LCR). En niños, la razón más común para realizar dicha práctica es llegar a diagnosticar una infección del sistema nervioso central, pero también es útil en el diagnóstico de otras enfermedades, así como realizar procedimientos terapéuticos en el caso de quimioterapia y antibióticos intratecales. ${ }^{3}$

The Royal College of Physicians and Surgeons of Canada (RCPSC) y el Accreditation Council for Graduate Medical Education (ACGME), señalan que las experiencias educacionales se deben enfatizar en competencias y habilidades para practicar pediatría general de alta calidad. Esto no sólo incluye conocimiento, si no también técnicas adecuadas en al menos 16 procedimientos, entre ellos la punción lumbar. ${ }^{4}$

Tradicionalmente, los médicos residentes han adquirido habilidades en la realización de una punción de ese tipo observando a médicos mayores o pediatras hacer el procedimiento varias veces y luego intentándolo ellos mismos, lo cual se asocia a la adquisición variable de la competencia ${ }^{3}$ y riesgos para los pacientes al presentar complicaciones asociadas al procedimiento, tales como dolor de espalda y cefalea postpunción lumbar con mayor frecuencia. Otras menos comunes incluyen dolor neurálgico de extremidades inferiores, hemorragia epidural, subdural o subaracnoidea, tumores raquídeos epidermoides, abscesos retroperitoneales, hipoxia. ${ }^{5}$

Muchos estudios han demostrado la falta de entrenamiento formal y la baja exposición a la punción lumbar durante la práctica clínica, lo cual conlleva a falta de confianza en sí mismo y una baja tasa de procedimientos exitosos, afectando la seguridad del paciente. ${ }^{1,3}$ Por lo anterior, muchos médicos están en riesgo de completar su residencia con una técnica y práctica inadecuadas del procedimiento.

Los programas de residencia en pediatría deberían focalizarse en asegurar adecuados métodos de enseñanza, supervisión y evaluación de habilidades en los procedimientos. ${ }^{1}$

Un estudio realizado en el programa de dicha especialidad en la Pontificia Universidad Católica de Chile (PUC), con base en encuestas de autopercepción, evidenció que más del 90\% de los residentes se sentía competente para realizar una punción de esta clase, sin mediar un programa de entrenamiento con simulación, a pesar de la gran variabilidad en cuanto al número de PL realizadas en el lapso de los tres años de formación (3 residentes realizaron 5 a $10 \mathrm{PL}, 2$ residentes 11 a $20 \mathrm{PL}, 4$ residentes más de $20 \mathrm{PL}) .{ }^{6} \mathrm{~A}$ pesar de todo lo anterior, si bien existen estudios de competencia en punción lumbar en pediatría, ${ }^{1}$ aún no se ha realizado una evaluación sobre la competencia en la realización de una punción semejante en un ambiente simulado. El siguiente estudio tiene por objetivo determinar la capacidad que tienen los residentes de esta especialidad médica, al final de su formación, para poder realizar 
una punción lumbar en un entorno simulado y comparar su desempeño con su autopercepción de competencia.

\section{MATERIAL Y MÉTODOS}

Se realizó un estudio descriptivo, con aprobación del Comité Ético Científico de Ciencias de la Salud de la Pontificia Universidad Católica de Chile. Durante los meses de noviembre y diciembre de 2019, se reclutó a todos los residentes de último año de un programa universitario de Pediatría de tres años de duración, que cumplieran con el criterio de inclusión de estar cercanos al término de su residencia. En noviembre de 2019 se aplicó una encuesta online donde se preguntó: 1. Autoevaluación de competencia en la realización de una punción lumbar basada en una escala de valoración global ordinal de cuatro categorías que va desde novato hasta experto, previamente validada para su uso por parte de evaluadores expertos, pero no en la evaluación de autopercepción ${ }^{7}$ (Tabla 1); 2. Número de punciones de este tipo realizadas a lo largo de la residencia; y 3 . Realización de punciones lumbares en ambiente simulado, especificando si fue mientras transcurría el pregrado en la universidad, pregrado fuera de la universidad y/o durante su residencia de Pediatría.

En diciembre de 2019, los residentes realizaron una punción de esta modalidad en el Centro de Simulación de la Pontificia Universidad Católica de Chile, habiendo firmado previamente un consentimiento informado.

En este procedimiento se utilizó el fantoma de Gaumard Scientific "Lumbar Puncture Skills Trainer S411" y se pusieron a disposición los siguientes

Tabla 1: Escala de evaluación global en punción lumbar pediátrica.

\begin{tabular}{|c|c|c|c|}
\hline Novato & Principiante & Competente & Experto \\
\hline $\begin{array}{l}\text { Orientación } \\
\text { extensa: más } \\
\text { de dos } \\
\text { instrucciones* }\end{array}$ & $\begin{array}{l}\text { Orientación } \\
\text { mínima: } \\
\text { una o dos } \\
\text { instrucciones* }\end{array}$ & $\begin{array}{l}\text { Realizada } \\
\text { de forma } \\
\text { independiente: } \\
\text { autocorrige, } \\
\text { comete errores } \\
\text { mínimos o } \\
\text { parece indeciso }\end{array}$ & $\begin{array}{l}\text { Realizada } \\
\text { de forma } \\
\text { independiente: } \\
\text { sin } \\
\text { instrucciones, } \\
\text { sin errores }\end{array}$ \\
\hline
\end{tabular}

* Instrucción se refiere a intervención verbal para prevenir o corregir un error. Adaptada al español desde Gerard JM, et al. ${ }^{7}$ materiales: alcohol gel para lavado de manos, gorros de procedimiento, mascarillas, guantes estériles, delantales estériles, bandeja regional, campo estéril perforado, alcohol $70^{\circ}$ para limpieza, ampolla de lidocaína al $2 \%$, jeringa $5 \mathrm{~mL}$, aguja $24 \mathrm{G}$, trocar espinal Pencan $25 \mathrm{G}$, tres tubos dedicados a toma de muestra. El procedimiento fue grabado en una sala del centro especializada con tal fin, sin la presencia de los evaluadores expertos. Los vídeos de cada participante fueron asignados con un número al azar para el posterior análisis por parte de dos evaluadores expertos en simulación de la misma institución pero que no formaban parte del programa de Pediatría durante los meses de septiembre y octubre de 2020. Cada uno evaluó los vídeos de forma independiente, basados en una escala de valoración global con la que también se midió la autopercepción de la competencia, a fin de poder realizar la comparación. La evaluación por vídeo de escalas de observación directa ha sido validada previamente por James M. Gerard y cols. ${ }^{7}$

La correlación entre observadores se realizó mediante el coeficiente weighted Kappa (wK); y su interpretación siguiendo lo propuesto por Landis y cols. ${ }^{8}$; con wK 0.00-0.20 como acuerdo escaso; 0.21-0.40 acuerdo aceptable; 0.41-0.60 acuerdo moderado; 0.61-0.80 acuerdo sustancial; y 0.811.00 acuerdo casi perfecto. Significancia estadística de $p<0.05$. El programa SPSS ${ }^{\circledR}$ versión 13 se utilizó para todos los análisis estadísticos.

\section{RESULTADOS}

Once de los doce residentes completaron la evaluación (92\%). En la autopercepción, todos los alumnos se consideraron a sí mismos competentes (7 de 11) o principiantes (4 de 11); ningún alumno se consideró novato o experto para la punción lumbar (Tabla 2). Los evaluadores expertimentados, en cambio, no evaluaron a ninguno de los estudiantes como experto ni competente. En las evaluaciones realizadas, los evaluadores presentaron un acuerdo sustancial (wK 0.62; $p<$ 0.01 ), siendo la mayoría considerados novatos (19 de 22) y pocos principiantes (3 de 22) (Tabla 3).

Todos los alumnos que fueron evaluados como principiantes se autopercibieron como competentes. Así, en todos los casos los estudiantes se autopercibieron más preparados que lo dicho por la evaluación experta, siendo la mitad de estas discrepancias con un nivel de diferencia entre las evaluaciones, y la otra mitad con dos niveles de diferencia entre ellas. El acuerdo entre 
Tabla 2: Resultados de encuesta aplicada a residentes.

\begin{tabular}{clcc} 
Residentes & Autoevaluación & $\begin{array}{c}\text { Número de } \\
\text { punciones } \\
\text { lumbares }\end{array}$ & $\begin{array}{c}\text { Simulación previa } \\
\text { en punción lumbar }\end{array}$ \\
\hline 1 & Competente & 8 & No \\
2 & Competente & 30 & Postgrado PUC \\
3 & Principiante & 10 & No \\
4 & Competente & 12 & No \\
5 & Principiante & 10 & No \\
6 & Competente & 15 & Pregrado \\
7 & Principiante & 8 & Pregrado \\
8 & Competente & 15 & No \\
9 & Competente & 3 & No \\
10 & Competente & 15 & No \\
11 & Competente & 4 & Pregrado y postgra- \\
12 & Principiante & 7 & do PUC \\
\hline
\end{tabular}

PUC $=$ Pontificia Universidad Católica de Chile. pediatría, independientemente del tipo de práctica clínica que elijan, se verán enfrentados en algún momento a pacientes que requieran una punción de estas características. ${ }^{9}$

Algunos estudios han intentado evaluar las competencias en PL de los residentes de Pediatría. Auerbach, en el año 2018, realizó un análisis descriptivo basado en una encuesta en línea que se aplicó a estudiantes de tercero y cuarto año de diez instituciones en los Estados Unidos, en la cual se preguntó información específica sobre la PL más recientemente realizada como la obtención de LCR, recuento de leucocitos, supervisión, lugar, uso de anestesia, uso de técnica de retiro temprano del estilete, familiar presente, ayudante para posicionar y sostener al paciente, además de su confianza en la realización y autoevaluación del procedimiento. La tasa de éxito de punción lumbar basado en el análisis de la última realizada fue de un 54\%, mientras que el $93 \%$ de los residentes se sentía confiado para realizar la intervención y 99\% se autoevaluó como competente en esta clase de punción en pacientes pediátricos, lo cual contrasta con la tasa de éxito mencionada anteriormente. En el presente trabajo se evidenció una situación similar, donde el $64 \%$ de los participantes se autoevaluó a nivel competente y el 36\% como principiantes, mientras que los evaluadores especialistas evaluaron a la mayoría (86\%) como novatos y sólo un $14 \%$ a nivel de principiantes, haciendo manifiesto el escaso grado de concordancia entre residentes y evaluadores expertos (wK 0.00; $p<0.01$ ) y la tendencia a autopercibirse más preparados en el procedimiento de lo que realmente están, como se exhibe también en la escasa literatura existente. Esto pudiera deberse a que las punciones lumbares realizadas en la práctica clínica no siempre se asocian a una adecuada supervisión ni retroalimentación posterior que buscara mejorar la adquisición de habilidades.

Por otra parte, varios reportes han demostrado la baja exposición a la punción lumbar durante la práctica clínica. ${ }^{1}$ Kilbane, en el año 2010, realizó un estudio intervencional prospectivo no aleatorizado, donde comparó un grupo experimental conformado por residentes de primer año (a quienes se les realizó una intervención educacional mediante simulación) y un grupo de control conformado por otros estudiantes de segundo año (sin la intervención educacional). Igualmente, intentaron evaluar las PL realizadas en pacientes en el Servicio de Urgencia infantil a lo largo de los seis meses que 
duró el estudio. Respecto a esto último, no hubo diferencias significativas entre ambos grupos pero sí destacó que la cantidad de PL realizadas fue muy baja, en promedio menos de una por residente, por lo que no fue posible sacar conclusiones en cuanto a las punciones lumbares efectuadas en el ambiente clínico, dada la baja exposición. ${ }^{4}$ En el mismo artículo de Auerbach del año 2018 mencionado anteriormente, se preguntó por el número de tales punciones llevadas a cabo, evidenciando que los residentes recién graduados efectuaron un promedio de 12 de dicha clase de punciones en el lapso de los cinco años de residencia, duración que dista de la realidad latinoamericana, con al menos una PL en los últimos tres meses. Esto se consideró un número limitado e insuficiente para el desarrollo y/o mantenimiento de las habilidades para una punción lumbar en pediatría. ${ }^{1}$ Algo similar se reveló en esta investigación, donde los participantes habían realizado un promedio de 11.41 PL durante los tres años de residencia pero con un amplio intervalo, ya que existían residentes que habían emprendido solo tres punciones lumbares mientras que otros habían consumado hasta 10 veces más intervenciones. Lo anterior pone en evidencia la baja exposición general al procedimiento, pero también la variabilidad extrema en cuanto a oportunidad de realización del procedimiento y por ende adquisición de habilidades en punción lumbar entre alumnos del mismo año y del mismo programa de Pediatría. Por otra parte, destaca que aquellos residentes más expuestos a PL no fueron mejor evaluados

\begin{tabular}{cccc} 
Tabla 3: Comparación entre residentes y evaluadores expertos. \\
Residentes & Autoevaluación & $\begin{array}{c}\text { Evaluador } \\
\text { experto } 1\end{array}$ & $\begin{array}{c}\text { Evaluador } \\
\text { experto } 2\end{array}$ \\
\hline 1 & Competente & Novato & Novato \\
2 & Competente & Novato & Novato \\
3 & Principiante & Novato & Novato \\
4 & Competente & Novato & Novato \\
5 & Principiante & Novato & Novato \\
6 & Competente & Novato & Principiante \\
7 & Principiante & Novato & Novato \\
8 & Competente & Novato & Novato \\
9 & Competente & No participa & No participa \\
10 & Competente & Novato & Principiante \\
11 & Competente & Novato & Principiante \\
12 & Principiante & Novato & Novato
\end{tabular}

por los expertos, lo que pudiera explicarse, como se comenta más arriba, debido a que muchos de esos procedimientos probablemente se realizaron sin retroalimentación posterior por parte del supervisor tratando de mejorar las competencias en el procedimiento.

En el estudio ya mencionado de Auerbach en 2018, también se les preguntó a los participantes sobre su exposición a entrenamiento en este tipo de punción a lo largo del primer año de residencia, donde un $73 \%$ afirmó haber recibido el adiestramiento pero no se preguntó si esto se había mantenido en el tiempo durante la residencia. En el presente escrito resultó patente que la mayoría de los residentes nunca había realizado una punción lumbar en ambiente simulado previamente y, de los que sí lo habían realizado, la mayoría lo había hecho en el transcurso del pregrado fuera de la universidad en la que se encontraban actualmente.

Este reporte refuerza la importancia de los simulacros que buscan aumentar el número de punciones realizadas durante la residencia, pero también para que éstas se desarrollen en un ambiente óptimo de aprendizaje, con entrenamiento deliberado de habilidades y retroalimentación efectiva.

Posibles futuras investigaciones serían evaluar la competencia de los residentes en punción lumbar previo y posterior a un programa de adiestramiento de simulación en dicho procedimiento, además de valorar si la autopercepción de competencias mejora, posterior al programa de entrenamiento simulado, pudiendo contrastar los resultados con los datos del presente estudio.

Dentro de las limitaciones de este trabajo destaca el posible sesgo de memoria respecto al número de punciones lumbares aplicadas por estos médicos, dado que se cuenta con un sistema de registro de procedimientos pero no todos los residentes lo mantienen actualizado.

\section{CONCLUSIONES}

Finalmente, los residentes se autoevalúan más competentes de lo que los expertos consideran. Al mismo tiempo, existe una gran variabilidad en cuanto a la exposición a punción lumbar durante la residencia, asociada a un insuficiente entrenamiento del procedimiento en simulación, lo cual evidencia la necesidad de que los programas de formación en Pediatría introduzcan el simulacro como un complemento a los métodos tradicionales para estandarizar y asegurar la adquisición 
de competencias en la ejecución de este tipo de punción.

Conflicto de intereses: El presente trabajo se realizó con el apoyo logístico (espacio e insumos) del Centro de Simulación de la Pontificia Universidad Católica de Chile. Los autores no tienen vínculos económicos ni recibieron aportes directos del Centro de Simulación. Los miembros del mencionado Centro no participaron en la escritura ni corrección del manuscrito.

\section{REFERENCIAS}

1. Auerbach MA, Lee WM, Bhargava S, Zaveri P, Seelbach EB, Burns RA, et al. Are graduating pediatric residents prepared to perform infant lumbar punctures? A multiinstitutional descriptive study. Pediatr Emerg Care. 2018; 34 (2): 116-120.

2. Vickers A, Donnelly JP, Moore JX, Barnum SR, Schein TN, Wang HE. Epidemiology of lumbar punctures in hospitalized patients in the United States. PLoS One. 2018; 13 (12): e0208622.

3. McMillan HJ, Writer H, Moreau KA, Eady K, Sell $\mathrm{E}$, Lobos AT, et al. Lumbar puncture simulation in pediatric residency training: improving procedural competence and decreasing anxiety. BMC Med Educ. 2016; 16: 198.

4. Kilbane BJ, Adler MD, Trainor JL. Pediatric residents' ability to perform a lumbar puncture: evaluation of an educational intervention. Pediatric Emerg Care. 2010; 26: 558-562.

5. De Gracia CP, De La Torre EM, Díaz M, García RS, Domínguez OG, Novoa CR. iSe realiza correctamente la punción lumbar en pediatría? Revisión de las recomendaciones actuales y análisis de la realidad. An Pediatr. 2012; 77 (2): 115-123.

6. Hirsch T, Castillo A, Katan J, Carrasco JA, Valle P, Triviño $X$. Procedimientos en el Programa de Especialidad de Pediatría: competencias necesarias y oportunidad de realizarlos. Presentado en congreso LACRE. 2013.

7. Gerard JM, Kessler DO, Braun C, Mehta R, Scalzo AJ, Auerbach M. Validation of global rating scale and checklist instruments for the infant lumbar puncture procedure. Simul Healthc. 2013; 8 (3): 148-154.

8. Landis JR, Koch GG. The measurement of observer agreement for categorical data. Biometrics. 1977; 33 (1): 159-174.

9. Ben-Isaac E, Keefer M, Thompson M, Wang VJ. Assessing the utility of procedural training for pediatrics residents in general pediatric practice. J Grad Med Educ. 2013; 5 (1): 88-92.

\section{Correspondencia:}

\section{Pablo Besa}

Pontificia Universidad Católica de Chile.

Marcoleta Núm. 367,

Santiago, Chile.

E-mail: pablobesa@gmail.com 